\title{
A simple mechanical analysis of the isentropic compression experiment
}

\author{
F. Montheillet ${ }^{1}$ and G. Roy ${ }^{2}$ \\ ${ }^{1}$ École Nationale Supérieure des Mines de Saint-Etienne, SMS-EMSE, CNRS UMR 5146, 158 cours Fauriel, \\ 42023 Saint-Etienne Cedex 2, France \\ ${ }^{2}$ CEA-Valduc, DRMN, 21120 Is-sur-Tille, France
}

\begin{abstract}
The uniaxial strain compression test, also referred to as isentropic compression experiment, was analyzed using a simple basically analytical model. It accounts for the compression under prescribed loading rate of a single phase elastic, elasticperfectly plastic, or elastic-viscoplastic material. In each case, stresses, elastic and plastic strain rates, as well as the kinetic, elastic, and plastic powers, were derived and compared.
\end{abstract}

\section{Introduction}

The isentropic compression experiment (ICE) has been increasingly used for the investigation of constitutive equations at very large strain rates and under high pressures. To a first approximation, it can be considered as a uniaxial strain compression test, where the loading rate can be prescribed for instance by an electromagnetic or laser impulse, or by detonation products stagnation $[1,2]$. In such conditions, the deformation of the specimen is both adiabatic (as far as the test duration is sufficiently short) and reversible, by contrast to the classical shock loading experiments. Such conditions are referred to as quasiisentropic, as plasticity is also likely to occur. A complete thermomechanical analysis of the experiment, including in particular inertia effects and wave propagation, obviously requires numerical computations. Nevertheless, the simple mechanical model proposed below allows to assess the influence of the various loading or material parameters straightforwardly. Purely elastic, elastic-perfectly plastic, and power law viscoplastic single phase materials will be considered.

\section{Uniaxial strain compression test}

\subsection{Geometry and assumptions}

Figure 1 shows schematically the uniaxial compression of a cylindrical specimen embedded in a non deformable die. The strains and strain rates can be considered as the sum of two components, viz. an elastic term (Fig. 1b), and a plastic or viscoplastic term (Fig. 1c), which may possibly be zero. The two velocity fields are assumed to be uniform at any time. This precludes wave propagation associated with dynamic loading, but still holds at large strain rates, as far as a material element (elementary representative volume) is considered. Therefore $\dot{\varepsilon}_{\mathrm{rr}}^{\mathrm{e}}=\dot{\varepsilon}_{\theta \theta}^{\mathrm{e}}$ and $\dot{\varepsilon}_{\mathrm{rr}}^{\mathrm{p}}=\dot{\varepsilon}_{\theta \theta}^{\mathrm{p}}$, for the elastic and plastic components, respectively, while the non-diagonal components are zero.

\subsection{Boundary conditions}

They are prescribed in terms of total velocity: $\dot{\mathrm{u}}_{\mathrm{r}}(\mathrm{R})=$ 0 , where $\mathrm{R}$ is the current specimen radius, at any point of the cylindrical boundary, $\dot{\mathrm{u}}_{\mathrm{z}}(0)=0$ on the lower horizontal surface of the specimen, and $\dot{\mathrm{u}}_{\mathrm{z}}(\mathrm{H})=\dot{\mathrm{H}}$ which is prescribed to the upper surface. More specifically, the following sinusoidal loading kinetics will be applied:

$$
\frac{\dot{\mathrm{H}}}{\mathrm{H}}=-\dot{\varepsilon}_{\mathrm{M}} \sin ^{2}\left(\frac{\pi}{2} \mathrm{t}_{\mathrm{n}}\right)
$$

where the normalized time $t_{n}=t / t_{M}$. At final time $\mathrm{t}_{\mathrm{M}}$, the loading rate reaches its maximum value $-\dot{\varepsilon}_{\mathrm{M}}$ (with $\dot{\varepsilon}_{\mathrm{M}}>0$ ). By adding and subtracting the two equations $\dot{\varepsilon}_{\mathrm{rr}}=\dot{\varepsilon}_{\mathrm{rr}}^{\mathrm{e}}+\dot{\varepsilon}_{\mathrm{rr}}^{\mathrm{p}}=0$ and $\dot{\varepsilon}_{\mathrm{zz}}=\dot{\varepsilon}_{\mathrm{zz}}^{\mathrm{e}}+\dot{\varepsilon}_{\mathrm{zz}}^{\mathrm{p}}=\dot{\mathrm{H}} / \mathrm{H}$, and taking into account plastic incompressibility, the two following relations are derived:

$$
2 \dot{\varepsilon}_{\mathrm{rr}}^{e}+\dot{\varepsilon}_{\mathrm{zz}}^{e}=\dot{\mathrm{H}} / \mathrm{H}=\dot{\mathrm{V}} / \mathrm{V} .
$$

where $\mathrm{V}$ denotes the volume of the specimen, and:

$$
\left(\dot{\varepsilon}_{\mathrm{rr}}^{\mathrm{e}}-\dot{\varepsilon}_{\mathrm{zz}}^{\mathrm{e}}\right)+\left(\dot{\varepsilon}_{\mathrm{rr}}^{\mathrm{p}}-\dot{\varepsilon}_{z z}^{\mathrm{p}}\right)=-\dot{\mathrm{H}} / \mathrm{H}
$$

Moreover, since $\dot{\varepsilon}_{\mathrm{rr}}^{\mathrm{p}}=-\dot{\varepsilon}_{\mathrm{rr}}^{\mathrm{e}}$, incompressiblity again implies $\dot{\varepsilon}_{\mathrm{zz}}^{\mathrm{p}}=2 \dot{\varepsilon}_{\mathrm{rr}}^{\mathrm{e}}$, which means that the (visco)plastic strain rates (and strains) can be immediately derived from their elastic counterparts, for any type of inelastic (incompressible) behaviour of the material.

\section{Purely elastic behaviour}

The material is first assumed isotropic and purely elastic. The Hooke equation:

$$
\sigma=(\kappa-2 \mu / 3) \operatorname{tr}\left(\varepsilon^{e}\right) \delta+2 \mu \varepsilon^{e}
$$

yields:

$$
\begin{gathered}
\sigma_{\mathrm{m}}=\frac{2 \sigma_{\mathrm{rr}}+\sigma_{\mathrm{zz}}}{3}=\kappa\left(2 \varepsilon_{\mathrm{rr}}^{\mathrm{e}}+\varepsilon_{\mathrm{zz}}^{\mathrm{e}}\right) \\
\sigma_{d}=\sigma_{\mathrm{rr}}-\sigma_{\mathrm{zz}}=2 \mu\left(\varepsilon_{\mathrm{rr}}^{\mathrm{e}}-\varepsilon_{\mathrm{zz}}^{\mathrm{e}}\right)
\end{gathered}
$$




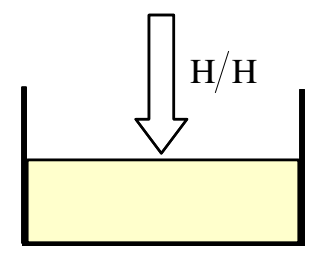

(a)

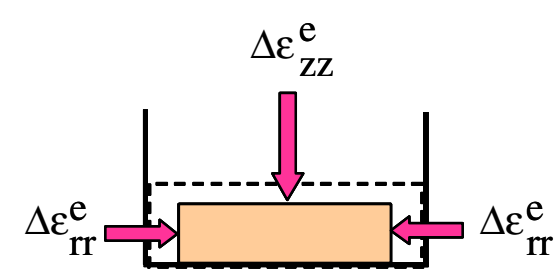

(b)

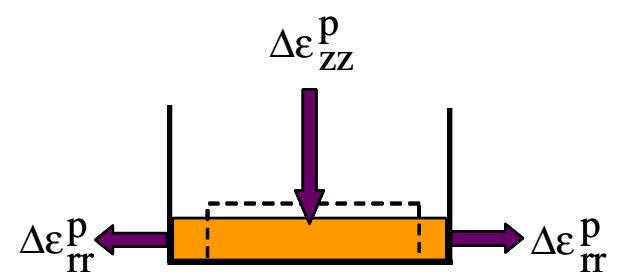

(c)

Fig. 1. Schematic view of the uniaxial strain $(I C E)$ compression test. (a) Initial state. (b) Elastic strain. (c) Plastic strain.

for the mean and deviatoric stresses, respectively. Assuming the elastic moduli $\kappa$ and $\mu$ remain constant during compression, and using Eqs. (2) and (3) after integration with respect to time gives:

$$
\sigma_{\mathrm{m}}=\kappa \ln \left(\mathrm{H} / \mathrm{H}_{0}\right)
$$

and:

$$
\sigma_{\mathrm{d}}=-2 \mu \ln \left(\mathrm{H} / \mathrm{H}_{0}\right)
$$

From the above equations, it is quite easy to derive the stress components $\sigma_{\text {rr }}$ and $\sigma_{z z}$, and the associated strains $\varepsilon_{\mathrm{rr}}^{\mathrm{e}}$ and $\varepsilon_{\mathrm{zz}}^{\mathrm{e}}$. In the case of a purely elastic material, closed form analytical expressions are thus obtained. It will be shown in the next section that it is the same in the case of the elastic-perfectly plastic behaviour.

\section{Elastic-perfectly plastic behaviour}

The plastic behaviour is assumed to be isotropic, according to the von Mises yield criterion and the associated flow rule. Whenever $\sigma_{\mathrm{d}}<\sigma_{0}$, where $\sigma_{0}$ is the von Mises flow stress, the analysis of 3 still holds. Plastic strain starts when $\sigma_{\mathrm{d}}=\sigma_{0}$, or, according to Eq. (8):

$$
\frac{\mathrm{H}}{\mathrm{H}_{0}}=\exp \left(-\frac{\sigma_{0}}{2 \mu}\right)
$$

In the elastic-plastic range, the mean stress is still given by Eq. (7), whereas $\sigma_{d}=\sigma_{0}$ remains constant. The stress and strain components then take the simple form:

$$
\begin{aligned}
& \left\{\begin{array}{l}
\sigma_{\mathrm{rr}}=\kappa \ln \left(\frac{\mathrm{H}}{\mathrm{H}_{0}}\right)+\frac{1}{3} \sigma_{0} \\
\sigma_{\mathrm{zz}}=\kappa \ln \left(\frac{\mathrm{H}}{\mathrm{H}_{0}}\right)-\frac{2}{3} \sigma_{0}
\end{array}\right. \\
& \left\{\begin{array}{l}
\varepsilon_{\mathrm{rr}}^{\mathrm{e}}=\frac{1}{3}\left(\ln \frac{\mathrm{H}}{\mathrm{H}_{0}}+\frac{\sigma_{0}}{2 \mu}\right) \\
\varepsilon_{\mathrm{zz}}^{\mathrm{e}}=\frac{1}{3}\left(\ln \frac{\mathrm{H}}{\mathrm{H}_{0}}-\frac{\sigma_{0}}{\mu}\right)
\end{array}\right.
\end{aligned}
$$

Figure $2 \mathrm{a}$ shows the time dependence of the stresses for $\kappa=140 \mathrm{GPa}, \mu=50 \mathrm{GPa}$, and $\sigma_{0}=1 \mathrm{GPa}$. For further comparisons the loading kinetics was imposed according to (1). It is worth to note, however, that time and strain rate have no influence on the behaviour in the present case. The (positive) deviatoric stress remains much less $\left(\sigma_{\mathrm{d}} \leq\right.$ $1 \mathrm{GPa}$ ) than the (negative, i.e. compressive) mean stress amplitude, such that the radial and axial stress components $\sigma_{\text {rr }}$ and $\sigma_{\mathrm{zz}}$ do not differ significantly.

Figure $2 \mathrm{~b}$ shows the associated elastic and plastic strains. In the elastic range, $\varepsilon_{\mathrm{rr}}^{\mathrm{e}}$ is zero due to the boundary conditions. By contrast, in the elastoplastic domain, two radial opposite strains $\varepsilon_{\mathrm{rr}}^{\mathrm{e}}<0$ and $\varepsilon_{\mathrm{rr}}^{\mathrm{p}}>0$ contribute to an overall zero strain, as illustrated in Fig. 1. During the first steps of plastic deformation, the modulus of $\varepsilon_{\mathrm{zz}}^{\mathrm{p}}$ is less than that of $\varepsilon_{\mathrm{zz}}^{\mathrm{e}}$, but the two curves cross at $\mathrm{t}_{\mathrm{n}} \approx 0.45$ and the plastic strain axial component becomes increasingly greater than the elastic one. This is also illustrated by Fig. 2c which shows the time dependence of the axial strain ratio $\mathrm{R}_{\mathrm{z}}=\varepsilon_{\mathrm{zz}}^{\mathrm{e}} / \varepsilon_{\mathrm{zz}}^{\mathrm{e}} \varepsilon_{\mathrm{zz}}^{\mathrm{p}}$, which is obviously infinite in the elastic range, then decreases monotonically, and tends to $1 / 2$ at large compression strains.

\section{Elastic-viscoplastic material}

The more general case of a nonlinearly elastic-viscoplastic material is now considered. Such kind of behaviour is illustrated in Fig. 3, which shows that the overall strain is the sum of an elastic component (spring) and a viscoplastic component (dashpot). For the sake of simplicity, the threshold $\sigma_{\mathrm{e}}$ (yield stress) will be set to zero in the following, and the viscoplastic behaviour will take the form of a power law:

$$
\sigma_{0}=k \dot{\bar{\varepsilon}}^{\mathrm{m}}
$$

where $\dot{\bar{\varepsilon}}$ is the von Mises equivalent strain rate and $\mathrm{m}$ the strain rate sensitivity parameter. When $\mathrm{m}=0$, this model is equivalent to the elastic-perfectly plastic case addressed in section 4 . When $\mathrm{m}=1$, the behaviour is elasticlinearly viscoplastic, which is tantamount for $\sigma_{\mathrm{e}}=0$, to the Maxwell viscoelastic model.

It is interesting to note that recent works have dealt with quite different constitutive equations $[3,4]$. In these approaches, the deviatoric stresses are first considered as negligible. Moreover, the mean stress is the sum of two terms: the first one is related to the elastic behaviour and 


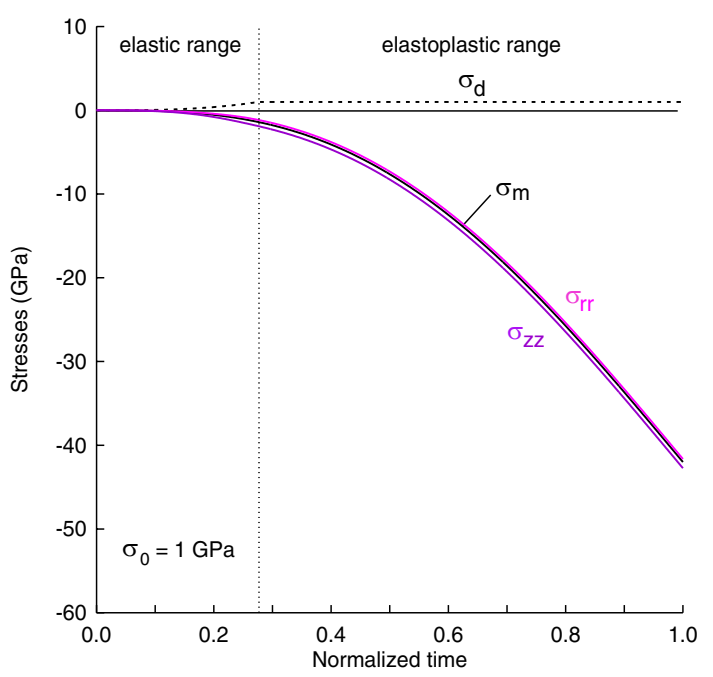

(a)

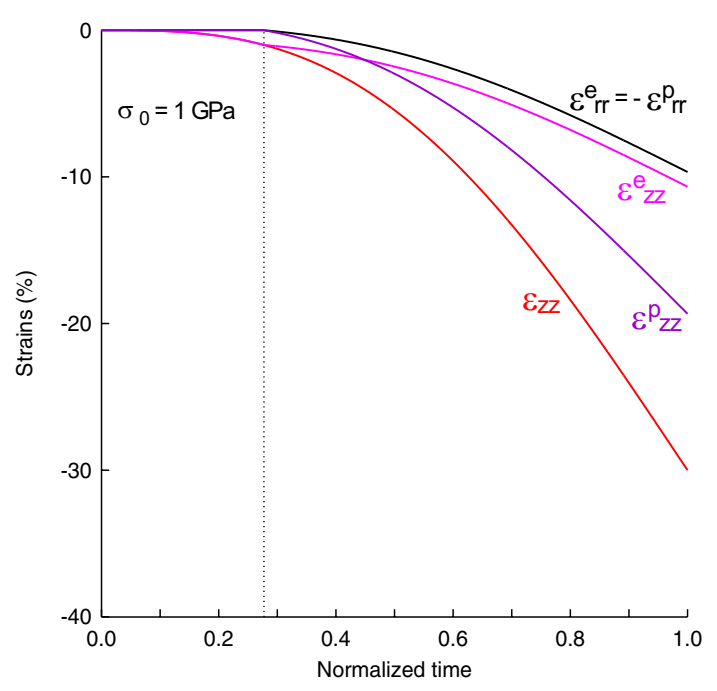

(b)

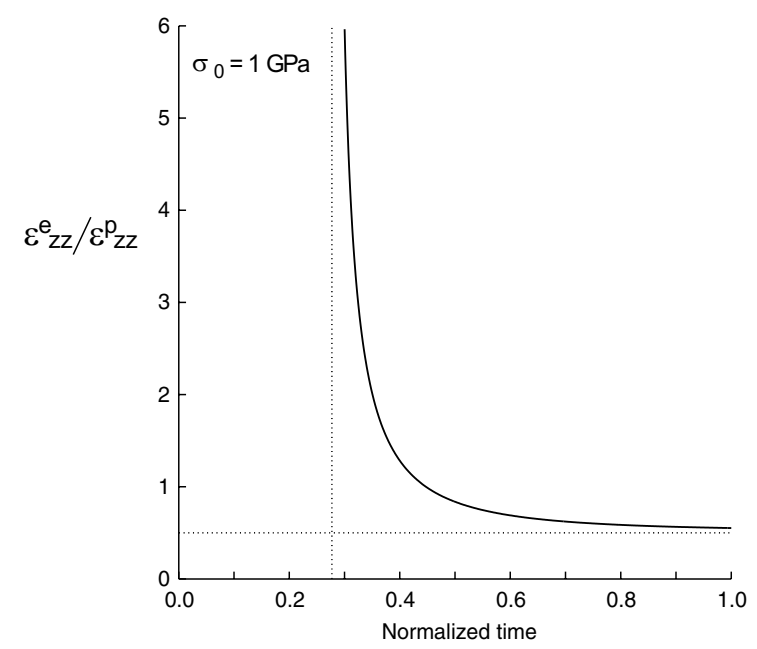

(c)

Fig. 2. Time dependence of the stresses (a), the strains (b) and the axial strain ratio (c) for an elastic-perfectly plastic material (see parameters in text)

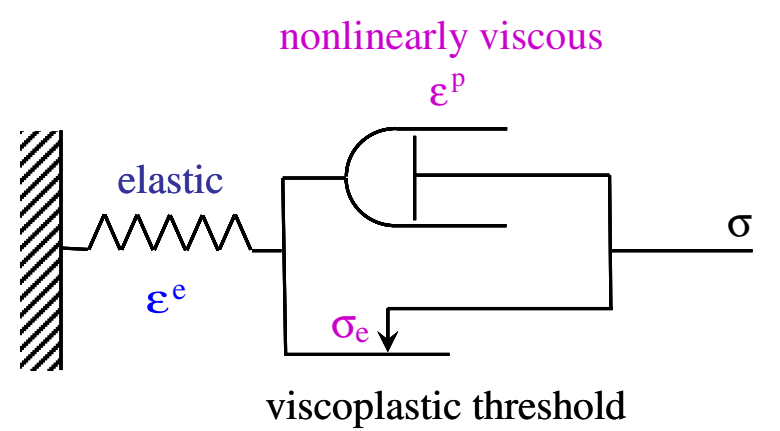

Fig. 3. Schematic representation of the elastic-viscoplastic behaviour.

the second reflects a strain rate sensitivity to the (elastic) volume change. By contrast, in the present model, viscous effects have a purely deviatoric origin.

The deviatoric stress $\mathbf{S}$ and plastic strain rate $\dot{\varepsilon}^{\mathrm{p}}$ tensors are related by the flow rule derived from the von Mises yield equation by the normality rule:

$$
\mathbf{s}=\frac{2}{3} \frac{\sigma_{0}}{\dot{\bar{\varepsilon}}} \dot{\varepsilon}^{\mathrm{p}}
$$

Since the strain rate tensor is diagonal, and taking into account cylindrical symmetry and plastic incompressibility, it is easy to show that $\dot{\bar{\varepsilon}}=2 \dot{\varepsilon}_{\mathrm{rr}}^{\mathrm{p}}=-2 \dot{\varepsilon}_{r r}^{e}$. Taking the inverse of Eq. (13) and using Eqs. (3) and (6) leads to the following differential equation for the unknown function of time $\sigma_{\mathrm{d}}$ :

$$
\dot{\sigma}_{\mathrm{d}}+\frac{3 \mu}{\mathrm{k}^{1} / \mathrm{m}}\left(\sigma_{\mathrm{d}}\right)^{1 / \mathrm{m}}=-2 \mu \frac{\dot{\mathrm{H}}}{\mathrm{H}}
$$

This equation must be solved numerically, except in the linear case $\mathrm{m}=1$. On the other side, $\sigma_{\mathrm{m}}$ is still given by Eq. (7), such that there is no coupling between deviatoric and volumic effects.

Equation (14) was solved for $\mu=50 \mathrm{GPa}, \mathrm{k}=$ $300 \mathrm{MPa}$, and various values of $\mathrm{m}$. The loading kinetics was given by Eq. (1), with $\dot{\varepsilon}_{\mathrm{M}}=10^{3} \mathrm{~s}^{-1}$ and $\mathrm{t}_{\mathrm{M}}=$ $6 \times 10^{-4} \mathrm{~s}$, leading to a final strain $\varepsilon_{\mathrm{M}}=-0,3$. For an initial thickness $\mathrm{H}_{0}=1 \mathrm{~mm}$ of the specimen, the final thickness is $\mathrm{H}_{\mathrm{M}}=0.74 \mathrm{~mm}$.

Figures $4 \mathrm{a}$ and $5 \mathrm{a}$ show the time dependence of the stresses for $\mathrm{m}=0.2$ and 1 , respectively. The deviatoric stress $\sigma_{\mathrm{d}}$, and thus the departure between the radial and axial stress components, significantly increase with $\mathrm{m}$. It is remarkable that Figs. $4 \mathrm{a}(\mathrm{m}=0.2)$ and $2 \mathrm{a}$ (elasticperfectly plastic material) look very similar: this illustrates the continuity of the mechanical behaviour when $\mathrm{m}$ decreases and tends to zero.

The corresponding elastic, plastic, and total strains are depicted in Figs. 4b and 5b. All are negative (compression), to the exception of the radial plastic component $\varepsilon_{\mathrm{rr}}^{\mathrm{p}}$ which is not shown in the diagrams. The amplitude of the axial plastic strain $\varepsilon_{\mathrm{zz}}^{\mathrm{p}}(<0)$ is much lower for $\mathrm{m}=1$ than $\mathrm{m}=0.2$, and the inverse is observed for the elastic axial component. For $\mathrm{m}=1$, the deformation of the material is therefore essentially elastic, whereas the plastic strain is predominant for $\mathrm{m}=0.2$. As for the stresses, comparison between figs. $4 \mathrm{~b}(\mathrm{~m}=0.2)$ and $2 \mathrm{~b}$ (elastic-perfectly plastic 


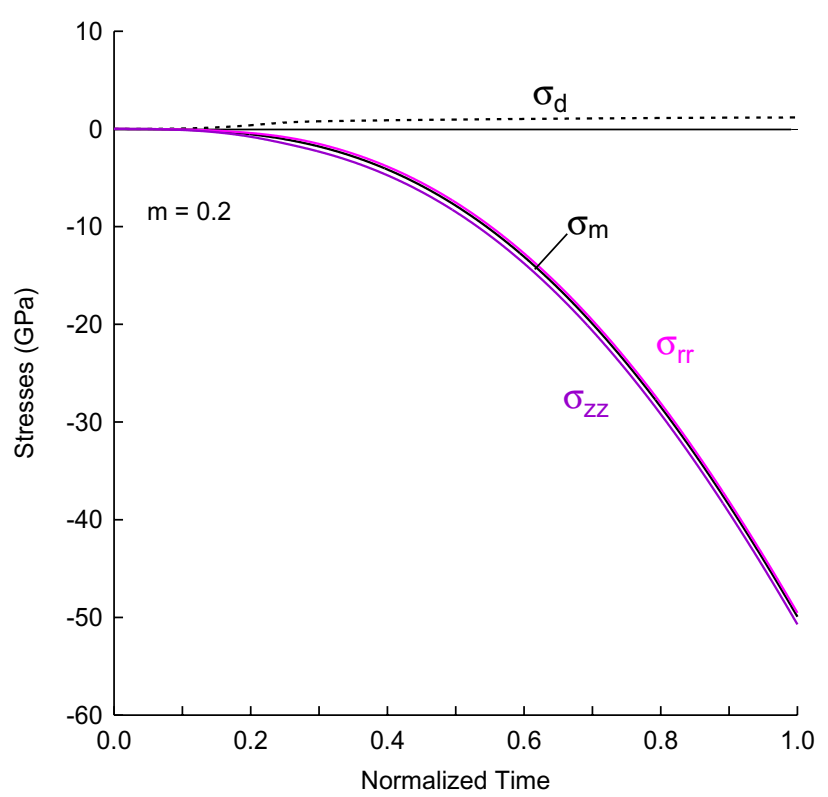

(a)

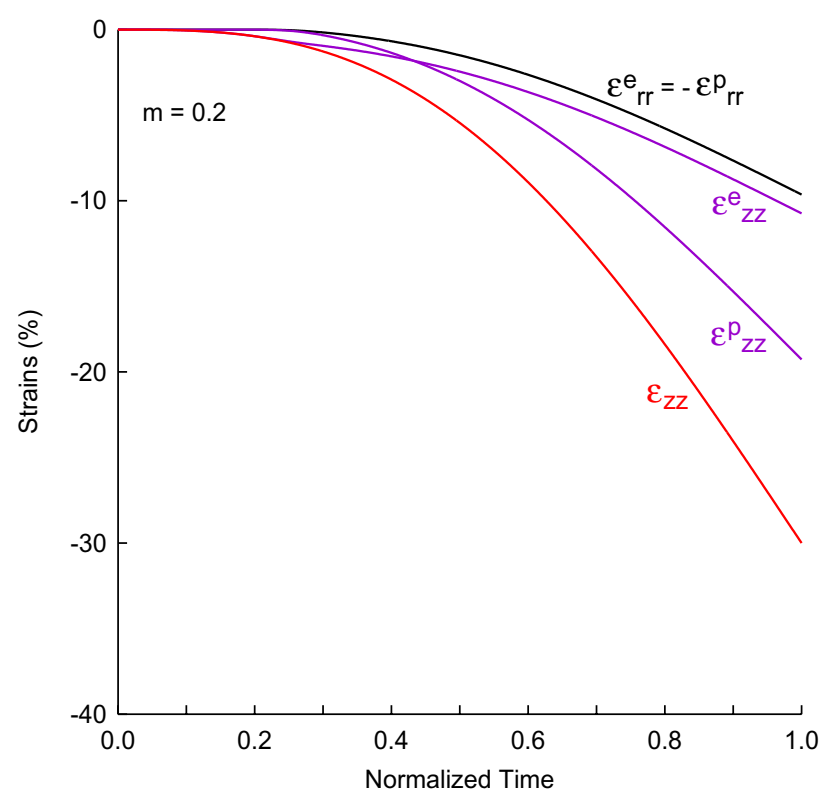

(b)

Fig. 4. Time dependence of the stresses (a) and the elastic, plastic, and total strains $(b)$ of a nonlinear $(\mathrm{m}=0.2)$ elastic-viscoplastic material.

material) shows the continuity of the material behaviour when $\mathrm{m} \rightarrow 0$.

Figure 6 depicts the time evolution of the $\mathrm{R}_{\mathrm{z}}$ ratio for $\mathrm{m}=0.2,0.5$, and 1 , which confirms the former remarks. Comparison with Fig. 2 shows a mathematical discontinuity of this dependence when $\mathrm{m} \rightarrow 0$ : whenever $m$ is nonzero, the initial value of $R_{z}$ is zero, whereas $R_{z}$ is infinite within the whole elastic range in the elastic-perfectly plastic case. Nevertheless, the mechanical

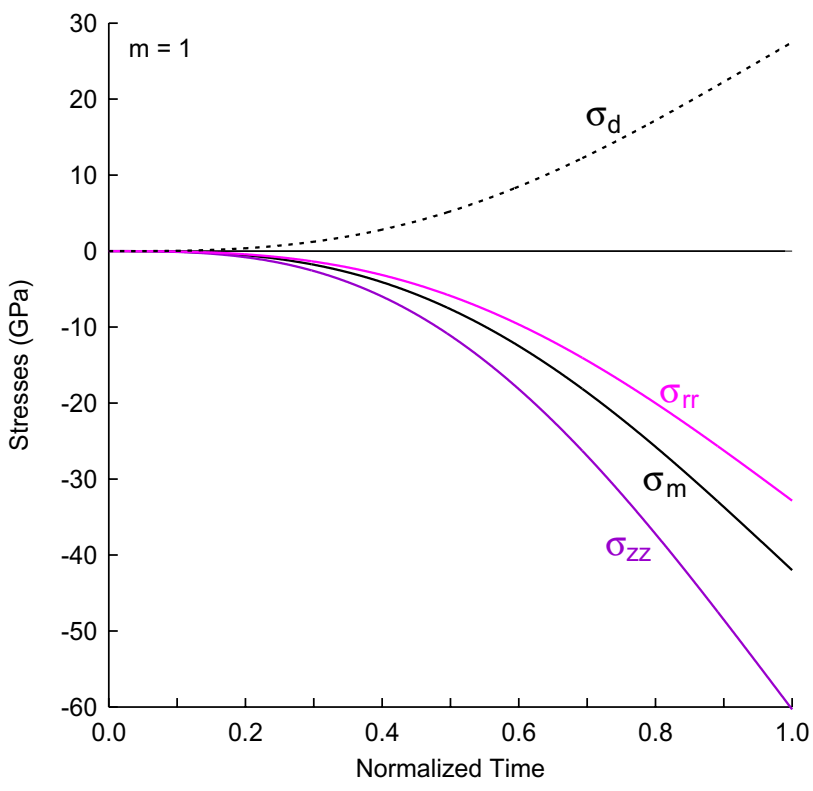

(a)

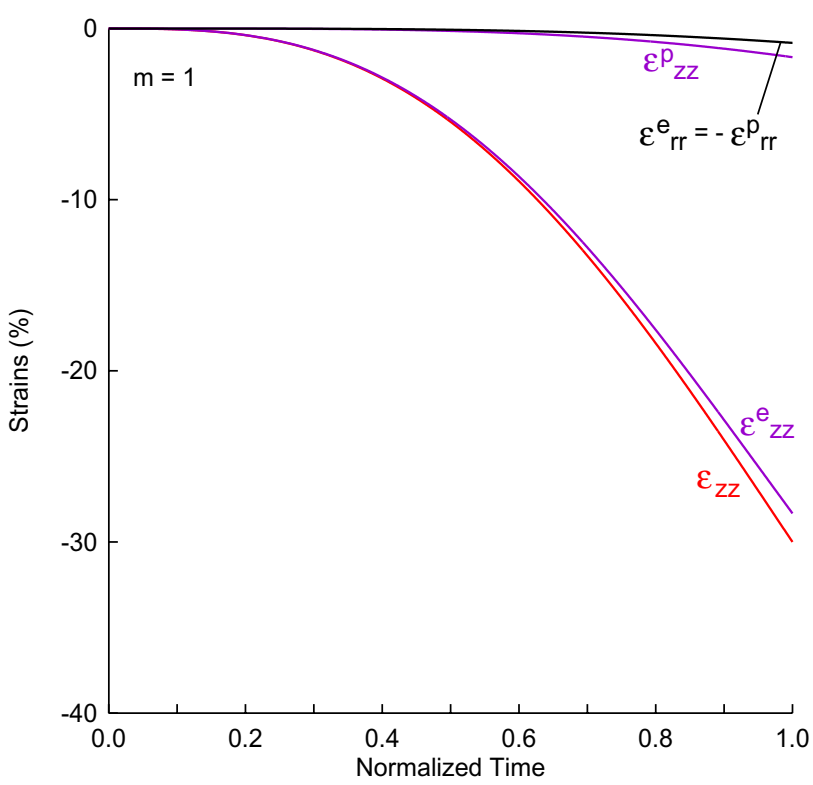

(b)

Fig. 5. Time dependence of the stresses (a) and the elastic, plastic, and total strains $(b)$ of a linear $(\mathrm{m}=1)$ elastic-viscoplastic material.

behaviour is consistent, since the initial peak of $R_{z}$ tends to infinity for $\mathrm{m} \rightarrow 0$.

\section{Kinetic, elastic, and plastic powers}

The present model allows to derive and compare the various powers involved in the isentropic compression experiment, associated with inertia effects, elastic, and plastic strain rates, respectively. 


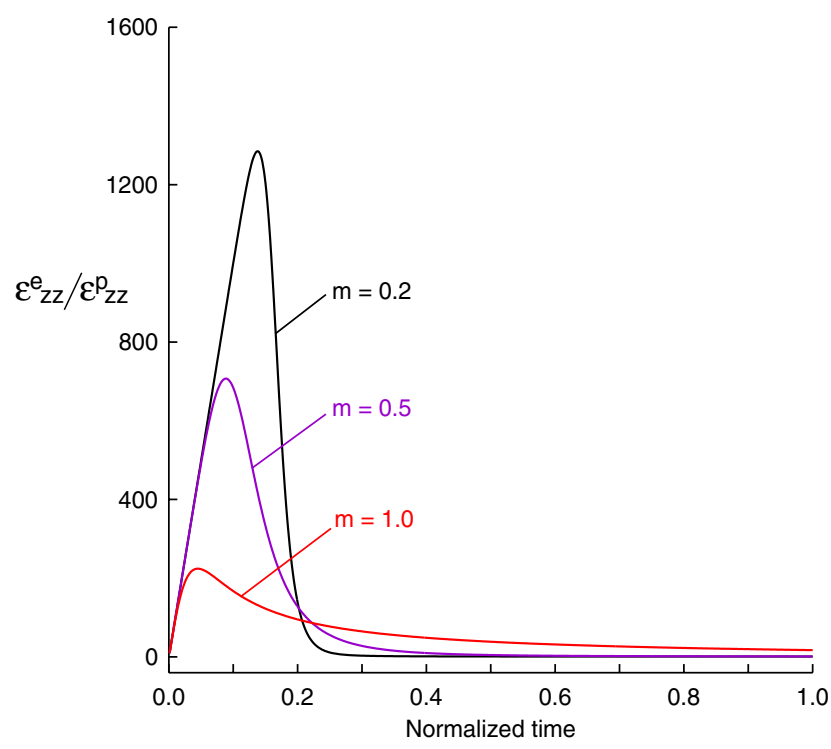

Fig. 6. Time dependence of the axial strain ratio for three elasticviscoplastic materials with $\mathrm{m}=0.2,0.5$, and 1 .

\subsection{Kinetic energy and power}

Assuming the axial velocity $\dot{\mathrm{u}}_{\mathrm{z}}$ varies linearly with $\mathrm{z}$ (uniform strain rate), and accounting for the mass conservation $\mathrm{M}=\rho_{0} \mathrm{~V}_{0}=\rho \mathrm{V}$, where $\mathrm{V}_{0}$ and $\mathrm{V}$ are the initial and current volumes of the specimen with densities $\rho_{0}$ and $\rho$, the kinetic energy can written in the form:

$$
\mathrm{E}_{\mathrm{c}}=\frac{1}{6} \mathrm{MH}^{2}
$$

Taking the time derivative yields the kinetic power:

$$
\dot{\mathrm{E}}_{\mathrm{c}}=\frac{1}{3} \mathrm{MH} \ddot{\mathrm{H}}
$$

where $\dot{H}$ and $\ddot{H}$ are given by Eq. (1). The time dependence of these two quantities is shown in Fig. 7 for a specimen of diameter $10 \mathrm{~mm}, \mathrm{H}_{0}=1 \mathrm{~mm}$, and an initial density $\rho_{0}=8960 \mathrm{~kg} \cdot \mathrm{m}^{-3} . \mathrm{E}_{\mathrm{c}}$ goes through a maximum near the end of the test (vertical dotted line), which merely reflects the loading kinetics Eq. (1).

\subsection{Elastic energy and elastic work rate}

Since the stresses are uniformly distributed within the sample, the current elastic energy can be written in the form:

$$
\mathrm{E}_{\mathrm{e}}=\left(\frac{\sigma_{\mathrm{m}}^{2}}{2 \kappa}+\frac{\sigma_{\mathrm{d}}^{2}}{6 \mu}\right) \mathrm{V}
$$

wherefrom the elastic work rate is merely obtained by taking the time derivative.

\subsection{Plastic work rate}

The plastic power per unit volume is given by the classical expression:

$$
\dot{\mathrm{w}}_{\mathrm{p}}=\mathrm{k} \dot{\bar{\varepsilon}}^{\mathrm{m}+1}
$$

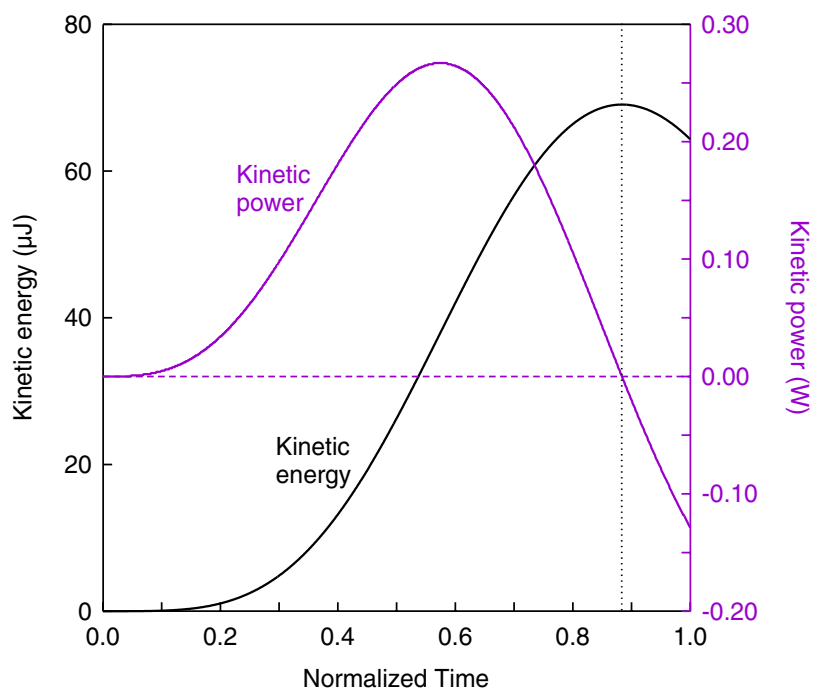

Fig. 7. Time dependence of the kinetic energy and kinetic power for a loading rate given by Eq. (1).

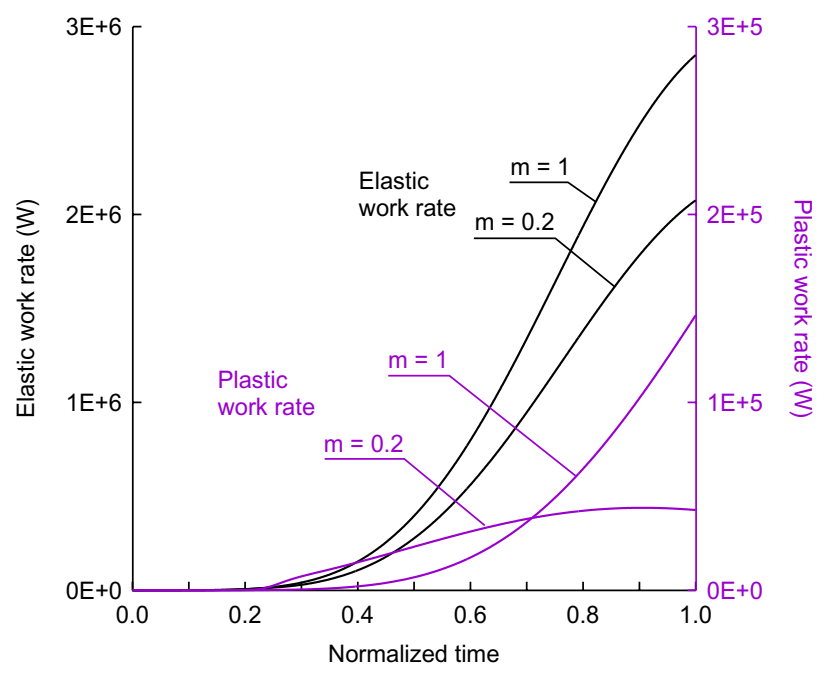

Fig. 8. Time dependence of the elastic and plastic work rates for elastic-viscoplastic materials with $\mathrm{m}=0.2$ and $\mathrm{m}=1$.

As shown above, the equivalent strain rate $\dot{\bar{\varepsilon}}=-2 \dot{\varepsilon}_{\mathrm{rr}}^{\mathrm{e}}$, which according to Eqs. (5) and (6) leads to:

$$
\dot{\bar{\varepsilon}}=-\frac{2}{3}\left(\frac{\dot{\sigma}_{\mathrm{m}}}{\kappa}+\frac{\dot{\sigma}_{\mathrm{d}}}{2 \mu}\right)
$$

whence:

$$
\dot{W}_{\mathrm{p}}=k\left[-\frac{2}{3}\left(\frac{\dot{\sigma}_{\mathrm{m}}}{\kappa}+\frac{\dot{\sigma}_{\mathrm{d}}}{2 \mu}\right)\right]^{\mathrm{m}+1} V
$$

Figure 8 compares the time dependence of $\dot{\mathrm{E}}_{\mathrm{e}}$ and $\dot{\mathrm{W}}_{\mathrm{p}}$ with the same parameters $\kappa, \mu$, and $\mathrm{k}$ as before, and two values of the strain rate sensitivity parameter $\mathrm{m}$. It appears that the time variations of these two powers is quite different according to the degree of viscosity of the material, which reflects the dissimilar partitions of the 
strains in the two cases (see Figs. 4 and 5). It is also worth to note that the plastic work rate is much smaller than its elastic counterpart: at the end of the test, $\dot{\mathrm{W}}_{\mathrm{p}} / \dot{\mathrm{W}}_{\mathrm{p}} \dot{\mathrm{E}}_{\mathrm{e}} \approx 0.02$ and 0.05 for $\mathrm{m}=0.2$ and $\mathrm{m}=1$, respectively. Finally, the kinetic power is negligible with respect to both above work rates (see Fig. 7).

\section{Conclusions and future work}

The uniaxial compression test, referred to as isentropic compression experiment (ICE) in the dynamic range, was investigated with an analytical (for elastic or elasticperfectly plastic materials) or semi-analytical (for elasticnonlinearly viscoplastic materials) model. A single phase material was addressed and strain was assumed to be uniform during the test. For each type of mechanical behaviour, the stress tensor, as well as the elastic, and (visco)plastic strain rate tensors, were derived as functions of the time under prescribed loading. The ratios of the elastic to plastic axial strains were also determined. All these parameters were shown to evolve in a consistent way within the range from perfectly plastic to linearly viscoplastic behaviour. It was also shown, using input data typical of a metallic material, that the kinetic power of the specimen is negligible with respect to the plastic work rate. Furthermore, the latter is itself much smaller than the elastic work rate.

In the near future, the model will be extended for investigating the mechanical behaviour of two phase elastic or viscoplastic materials. In particular, the complex interactions coupling deformation and phase transformation will be addressed using various homogeneization assumptions for the mixture properties. Moreover, comparisons will be carried out with experimental data.

\section{References}

1. J.-P. Davis, Ch. Deeney, M.D. Knudson, R. Lemke, T.D. Pointon, D.E. Bliss, Phys. Plasmas 12, 056310, p. 1-7 (2005)

2. J.R. Asay, T. Ao, J.-P. Davis, C. Hall, T.J. Vogler, G.T. Gray III, J. Appl. Phys. 103, 083514, p. 1-16 (2008)

3. J.L. Ding, J. Mech. Phys. Solids 54, 237 (2006)

4. J.L. Ding, J.R. Asay, J. Appl. Phys. 101, 073517, p. 119 (2007) 